journal club

\title{
Negativsymptomatik wird nicht beeinflusst
}

Fragestellung: Kann eine Körperpsychotherapie im Gruppensetting (KPG) die Negativsymptomatik bei Patienten mit einer Schizophrenie verbessern?

Hintergrund: Die Schizophrenie stellt eine schwere psychische Erkrankung dar, von der etwa 0,7\% der Bevölkerung betroffen sind. In der akuten Phase zeigen sich vornehmlich Positivsymptome, die allgemein Übersteigerungen und Fehlinterpretationen der Wahrnehmung oder des Erlebens beschreiben. Die Negativsymptomatik hingegen umfasst Defizite im Ausdrucksverhalten. Hierzu gehören sozialer Rückzug, Affektverflachung und Sprachverarmung. Negativsymptome nehmen starken Einfluss auf den Langzeitoutcome. „Art therapies“, unter denen nonverbale Therapieformen wie Kunsttherapie, Musiktherapie oder KPG verstanden werden, wurden durch das NICE (National Institute for Health and Care Excellence) in Großbritannien als einzige wirksame Behandlungsansätze für diese Symptomatik empfohlen. Trotz dieser Empfehlung fehlte bislang eine ausreichende Evidenz.

Priebe S, Savill M, Wykes T et al. Effectiveness of group body psychotherapy for negative symptoms of schizophrenia: multicentre randomised trial. Brit J Psychiatry 2016; 209: 54-61
Patienten und Methodik: Ambulante Patienten mit einer Schizophrenie (definiert durch ICD Code F20.0 bis F20.9) zwischen 18 und 65 Jahren, einem Wert von $\geq 18$ in der Negativskala der
PANSS (Positive and Negative Syndrome Scale), ohne Veränderung der antipsychotischen Medikation seit sechs Wochen, und der Bereitschaft, aktiv an den Gruppeninteraktionen teilzunehmen, wurden in die Studie eingeschlossen. Die klinische Studie war multizentrisch, randomisiert, verblindet und aktiv kontrolliert. Neben der Standardversorgung nahmen die Patienten entweder an KPG oder Pilates teil. Der Endpunkt wurde vor Beginn, am Ende sowie sechs Monate nach Beendigung der Therapie evaluiert. Der primäre Endpunkt entsprach dabei dem PANSS-Wert der Negativskala am Therapieende; zusätzlich wurden noch sekundäre Endpunkte erhoben.

Ergebnisse: Von 275 in die Studie eingeschlossenen Patienten verblieben 255 bis zur Follow-up-Untersuchung sechs Monate nach Therapieende. Zu Beginn lag der PANSS-Wert der Negativskala in der KPG-Gruppe bei 23,3 und in der Pilatesgruppe bei 23,1. Beide Gruppen zeigten eine geringfügige Abnahme des PANSS vom Zeitpunkt vor der Studie bis zum Therapieende und der Follow-up-Untersuchung sechs Monate nach Therapieende. Hierbei bestand jedoch kein signifikanter Gruppenunterschied hinsichtlich des PANSS-Werts in der Negativskala zum Therapieende $(21,8$ bzw. $21,5, \mathrm{p}=0,959)$ sowie sechs Monate später (21,7 bzw. 21,7, $\mathrm{p}=0,812)$.

Schlussfolgerung: Die KPG hat keinen klinisch relevanten Einfluss auf die Negativsymptomatik von Patienten mit Schizophrenie.

\section{- Kommentar von Lea Christina Bensing und Nadine Dreimüller, Mainz}

\section{Therapie bei Negativsymptomatik - welche Möglichkeiten gibt es?}

Die methodisch hochwertige Studie (Evidenzstufe II) hat aufgrund des Designs 4 von 5 Jadad-Punkten verdient. Insbesondere ist hervorzuheben, dass es sich um eine aktiv kontrollierte Studie handelt. Jedoch muss man feststellen, dass die Ergebnisse die Empfehlungen des NICE infrage stellen. Da es im Unterschied zur Positivsymptomatik nur wenige effektive Konzepte zur Behandlung der Negativsymptomatik gibt, stellt diese Publikation trotz nicht signifikanter Ergebnisse einen positiven Beitrag dar. Kritisch zu beurteilen ist, dass die Patienten im Mittel mit einem Wert von etwa 23 eher mäßig beeinträchtigt waren. So zeigen Studien zur Bewertung von Therapieansätzen der Depression, dass schwer beeinträchtigte Patienten von Interventionen mehr profitieren. Ein analoges Muster könnte auch in dieser Studie vorliegen.

Vorangegangene Arbeiten legen nahe, dass psychologische Faktoren wie etwa Bewertungen und bestimmte Informationsverarbeitungsprozesse Einfluss auf die Negativsymptomatik nehmen. So hängt Negativsymptomatik unter anderem mit Schwierigkeiten in der "Theory of Mind" (weniger Empathie, geringerem Selbstwert und negativeren Selbstkonzepten) sowie dysfunktionalen Annahmen zusammen [1]. Da die Mög- lichkeiten von Interventionen auf den Ebenen von Sprache (z. B. formale Denkstörungen) und Emotionen (z. B. Anhedonie) bei dieser Patientengruppe aufgrund der beschriebenen Symptomatik deutlich eingeschränkt sind, liegt ein Therapiekonzept nahe, dass zwar körperbezogen ist, aber zudem oben genannte wirkungsvolle Faktoren der sozialen Kognition berücksichtigt.
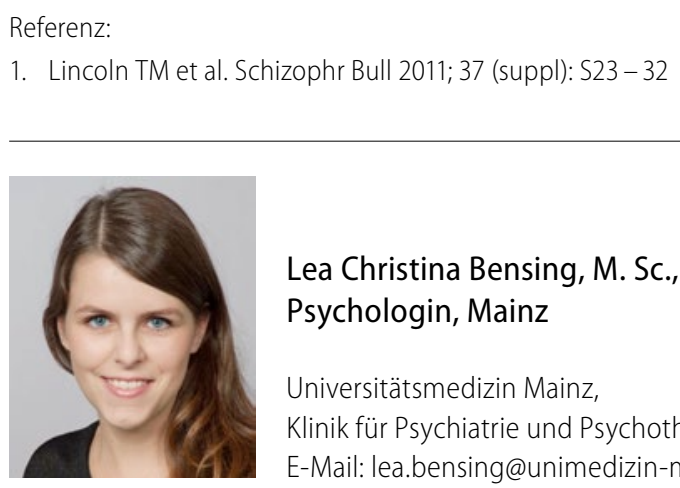

Lea Christina Bensing, M. Sc., Psychologin, Mainz

Universitätsmedizin Mainz, Klinik für Psychiatrie und Psychotherapie E-Mail: lea.bensing@unimedizin-mainz.de 